\title{
On MLGP- Rings
}

\section{Raida D.mahmood}

Ebtehal S. Mageed

raida.1961@uomosul.edu.iq

College of Computer Sciences and Mathematics

University of Mosul, Mosul, Iraq

\begin{abstract}
An ideal $K$ of a ring $R$ is called right (left) generalized pure ( $G P$-ideal)if for every $a \in K$, there exists $m \in Z^{+}$, and $b \in K$ such that $a^{m}=a^{m} b\left(a^{m}=b a^{m}\right)$. A ring $R$ is called $M L G P$ - ring if every right maximal ideal is left $G P$-ideal . In this paper have been studied some new properties of $M L G P$ - rings and the relation between this rings and strongly $\pi$ - regular rings some of the main result of the present work are as follows:

1- Let $R$ be a local , MLGP and $S X M$ ring. Then :

(a) $J(R)=0$.

(b) If $R$ is $N J$ - ring. Then $r\left(a^{m}\right)$ is a direct sum and for all $\in R, m \in Z^{+}$.

2 - Let $R$ be a local , $S X M$ and $N J$ - ring. Then $R$ is strongly $\pi$-regular if and only if $R$ i $L G P$.
\end{abstract}

Keywords: NJ Rings , SXM rings ,local strongly regular rings ,pure ideals

$$
\text { MLGP- حول الحلقات من النمط }
$$

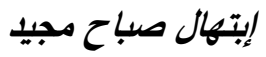

رائدة داؤد محمود

$$
\text { جلية علوم الحاسوب والرياضيات الريات الدوصل، الدوصل، العراق }
$$

تاريخ قبول البحث: 2019106130

تاريخ استلام البحث: $2019 \backslash 06118$

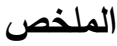

يقال للمثالي K في الحلقة R ، بأنه مثالي نقي معمم أيمن (أيسر) و اختصاراً من النمط ـ GP إذا كان لكل Kوجد عدد صحيح موجب $m$ و حلقة من النمط ـ MLGP إذا كان كل مثالي أعظمي أيمن هو مثالي من النمط ـ GP أيسر ـ قدمنا في هذا البحث

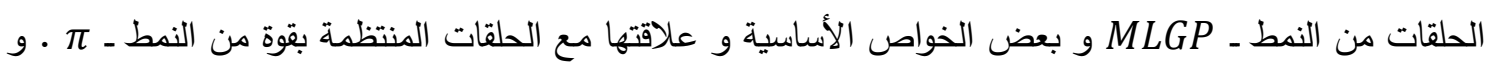
من أبرز النتائج التي حصلنا عليها :

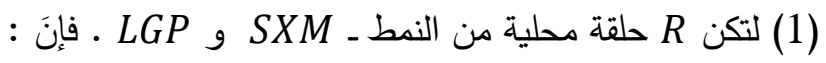
. $J(R)=0(\mathrm{a})$

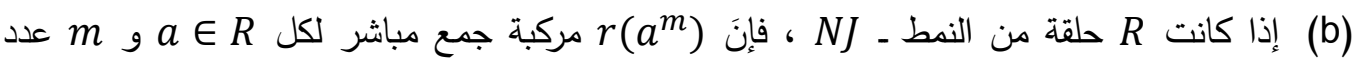




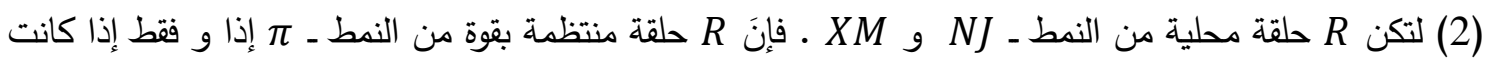

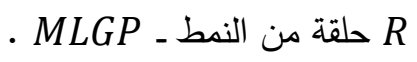
الكلمات المفتاحية: حلقات NJ ، حلقات SXM ، حلقات محلية منتظمة بقوة ، مثاليات نقية.

\section{1.المقدمة Introduction}

في هذا البحث كل حلقة R تجميعية بعنصر محايد ـ يقال للمثالي K في الحلقة R بأنه مثالي من النمط ـ

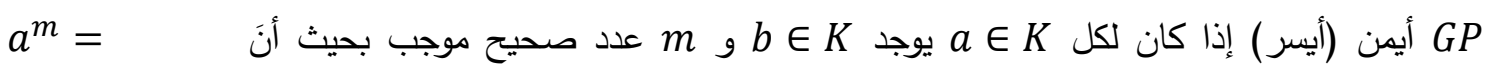

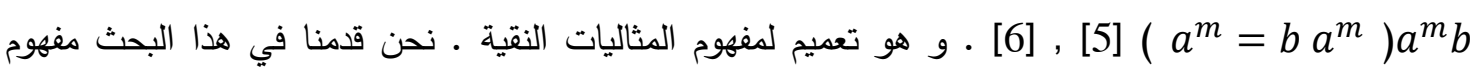

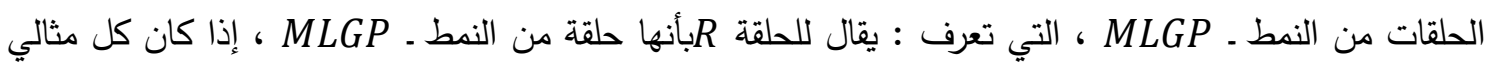

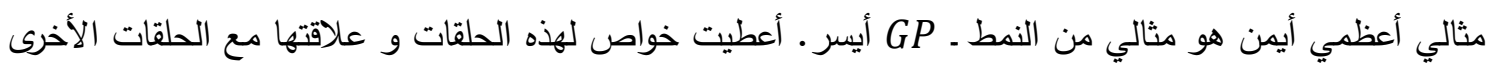
• لكل ( رمز مجموعة العناصر المعدومة القوى ، و جذر جاكوبسون و المثالي المنفرد الأيمن (الأيسر) التوالي

يقال للحلة R بأنها من النمط ـ NJ ، إذا كان (R)

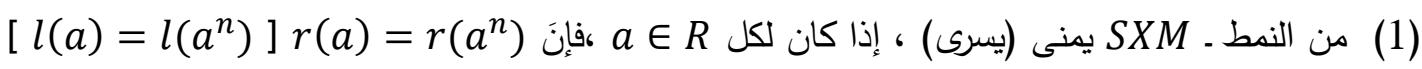

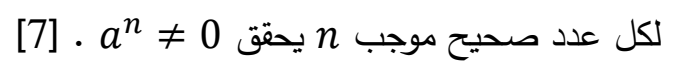

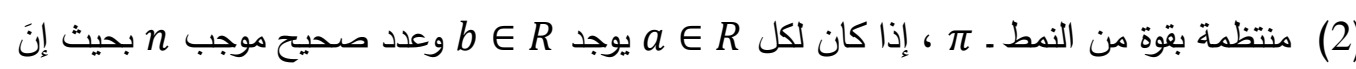

$$
\text { [5] } \cdot\left(a^{n}=a^{2 n} b\right)
$$

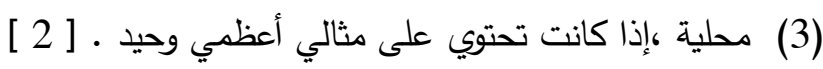

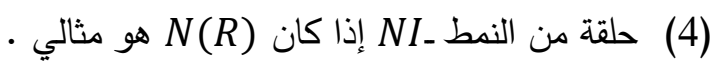

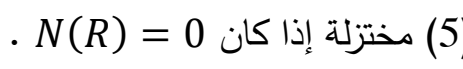

MLGP _ الحلقات من النمط

ندرس في هذا البند المثاليات من النمط ـ GP و الحلقات من النمط ـ MLGP و بعضاً من خواصها الأساسية

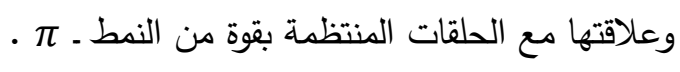

\section{مبرهنة 2.1 :}

لتكن R حلقة إبدالية ، فإن التقاطع المنتهي للمثاليات من النمط ـ GP هو مثالي من النمط ـ GP.

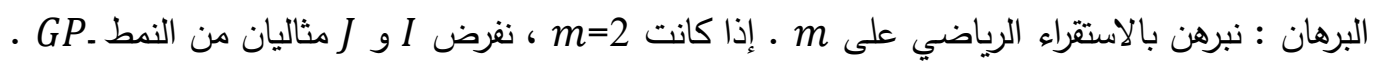
( القضية 3.1 .4 [5] ) ، فإن J I I مثالي من النمط ـ GP ـ نفترض أن القضية صحيحة ل m من المثاليات

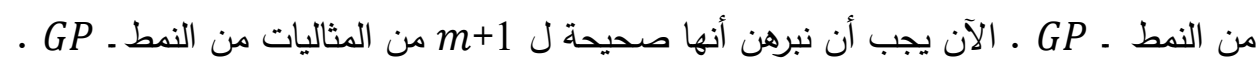
ليكن

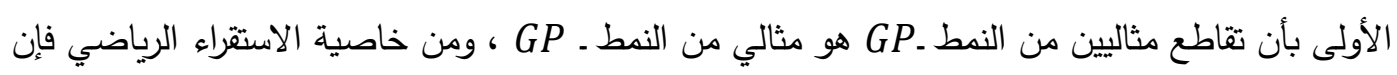

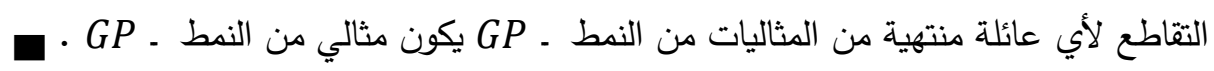


لتكن R حلقة من النمط ـ N N ، و كل مثالي رئيس هو مثالي أيمن من النمط ـ GP ، فإن

$$
\text { . } J(R)=N(R)
$$

البرهان : نفترض أن (R)

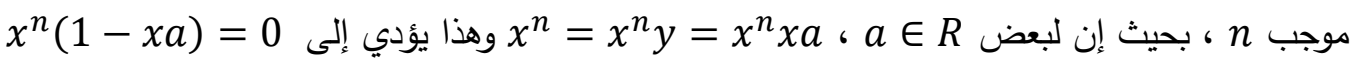

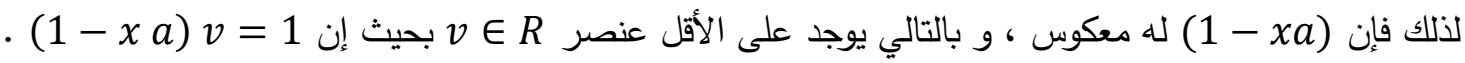

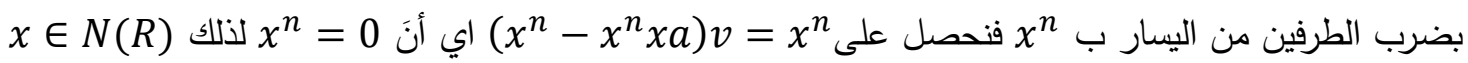

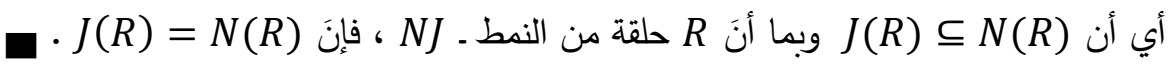
قضية 2.3 : (2.

لتكن R حلقة حيث إن كل مثالي رئيس فيها هو مثالي من النمط ـ GP أيمن ، فإن العبارات الآتية متكافئة : . (1)

م متتاظرة بضعف .

$$
\text { . } N(R)=J(R)
$$

مI ملقة من النمط - RI

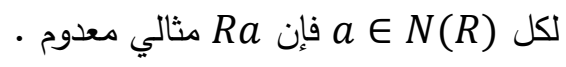

. NJ - R/J(R) حلقة من النمط

. NJ - ملقة من النمط

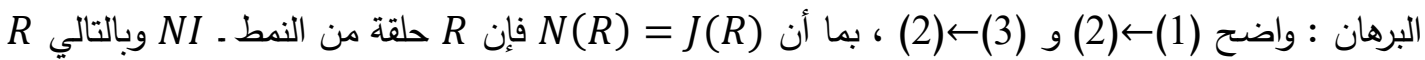

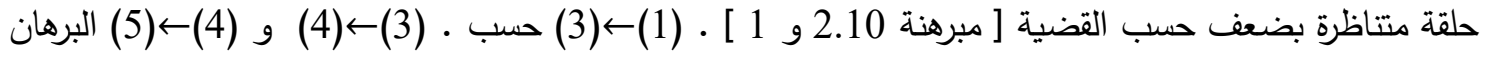

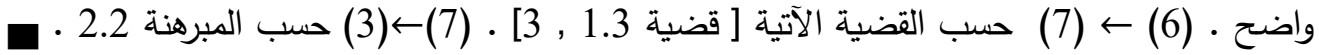
الآن نعطي التعريف الآتي :

يقال للحلقة R بأنها حلقة من النمط ـ MLGP إذا كان كل مثالي أعظمي أيمن هو مثالي من النمط ـ GP $I=\left\{\left[\begin{array}{ll}1 & 1 \\ 0 & 0\end{array}\right],\left[\begin{array}{ll}1 & 0 \\ 0 & 0\end{array}\right],\left[\begin{array}{ll}0 & 1 \\ 0 & 0\end{array}\right],\left[\begin{array}{ll}0 & 0 \\ 0 & 0\end{array}\right]\right\}$ مثال : لتكن $R=\left\{\left[\begin{array}{ll}x & y \\ z & 0\end{array}\right]: x, y, z \in Z_{2}\right.$ مثالي أعظمي أيمن وهو مثالي من النمط . GP أيسر، بالتالي فإن R حلقة من النمط ـ MLGP . 
لتكن R حلقة محلية من النمط ـ SX و MLGP فإنَ :

$$
\cdot J(R)=0
$$

(2) إذا كانت R حلقة من النمط ـ NJ ، فإنَ (a (a) مركبة جمع مباشر لكل a

البرهان : (1) بما أن R حلقة محلية ، (R) Jو مثالي أعظمي أيمن وحيد في R [4] أي أن (R) J Jثالي من النمط ـ GP بالتالي فإن لكل (1) بما أن (R) . $J(R)=0$ = 0

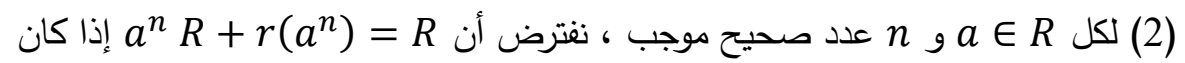

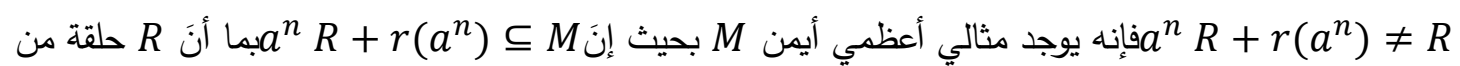

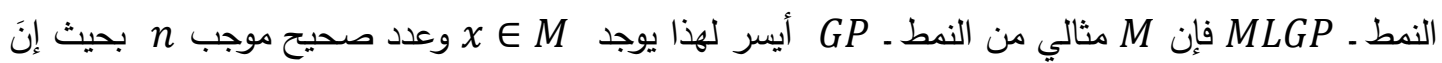

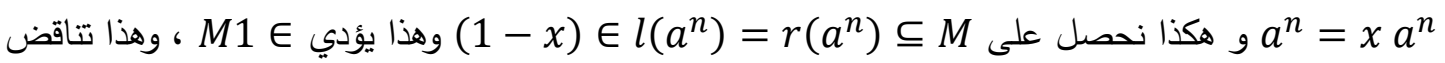
إذاً

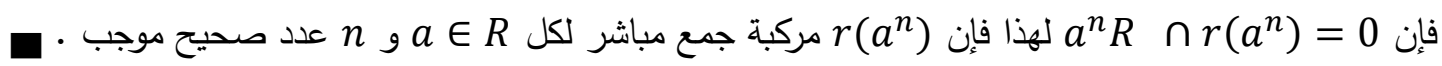
نتيجة 2.6 : لتكن R حلقة محلية من النمط ـ SXM ، NJ و MLGP ، فإن .

$$
\text { . } Y(R)=Z(R)=0(2)
$$

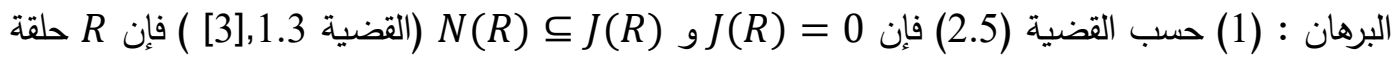

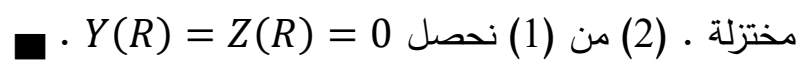

من الواضح أنَ كل حلقة منتظمة بقوة من النمط ـ $\pi$ هي حلقة من النمط ـ MLGP ، لكن العكس غير صحيح . المبرهنة الآتية تعطي الثرط الكافي في الحلقة MLGP لتكون حلقة منتظمة بقوة من النمط ـ $\pi$ ـ

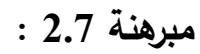

لتكن R حلقة محلية من النمط ـ NJ و SXM ـ فإن R حلقة منتظمة بقوة من النمط ـ $\pi$ إذا وفقط إذا كانت R حلقة من النمط ـ MLGP

البرهان : نفترض أنَ لكل a $a \in R$ يوجد عدد صحيح موجب n بحيث إنَ

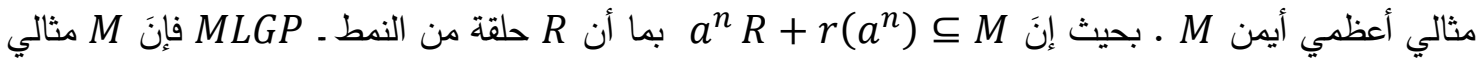


من النمط ـ GP أيسر لذلك يوجد $x \in M\left(a^{n}\right)^{m}$ و عدد صحيح موجب بحيث إنَ

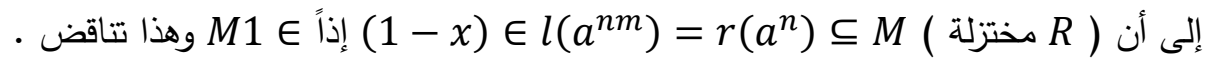

لذلك فإنَ

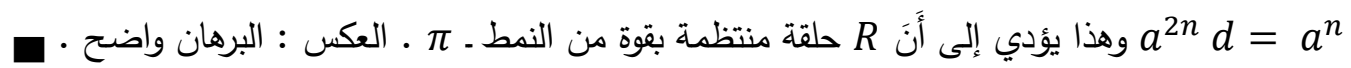

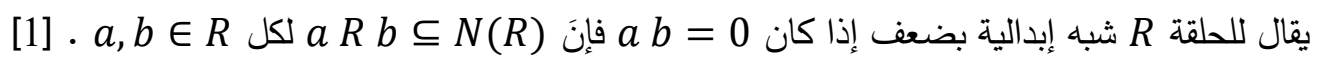

المبرهنة الآتية تبين متى تكون الحلقة الثبه إبدالية بضعف حلقة مختزلة .

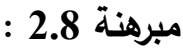

لتكن R حلقة محلية من النمط ـ SXM و MLGP ، فإن R حلقة شبه إبدالية بضعف إذا وفقط إذا كانت

R ملقة مختزلة .

البرهان : نفترض أنَ R حلقة غير مختزلة عند ذلك يوجد

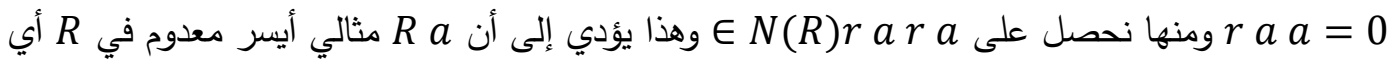

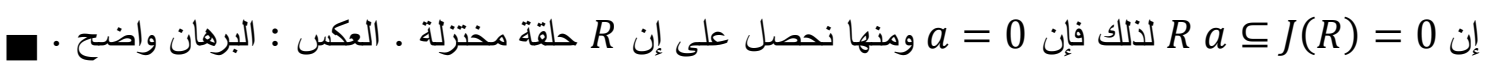




\section{المصادر}

[1] Abdullah , H. Handan , K. and Bureu , U. (2018 ), " On Weak symmetric property of rings", Sou . Asian, Bull . of Math . 42, pp 31-40 .

[2] Burton , D.M. (1970) ; "AFirstcourseinRingsandideals", AddisonWeslypublishing .

[3] Chang ,L. and Soo, Y. p. (2018) " When nilpotents are contanined in Jacobson radicals " J . Korean . Math . Soc . 55 , No .5, pp , 1193-1205 .

[4] Hazewinkel , M . , Gubareni , N . and Kiriehenko V. V. , (2004) , " Algebras , Rings and Modules " Vol .1 Kluwer Academic publishers .

[5] Mahmood , R. D. (2000), "Onpureidealsandpuresubmodule When nilpotents are contanined in Jacobson radicals " , Ph .D. , Thesis, Mosuluniversity .

[6] Mahmood, R. D. and Mahmood ,A. B. (2008), " Maximal generalization of pure ideals " Raf . J . of comp . and Math ,Vol . 5 , N1 , pp, $21-27$.

[7] Wei , J. C. (2007), " On simple singular YJ-injective modules " , Sou. Asian Bull . Of Math. 31, pp.1-10 . 